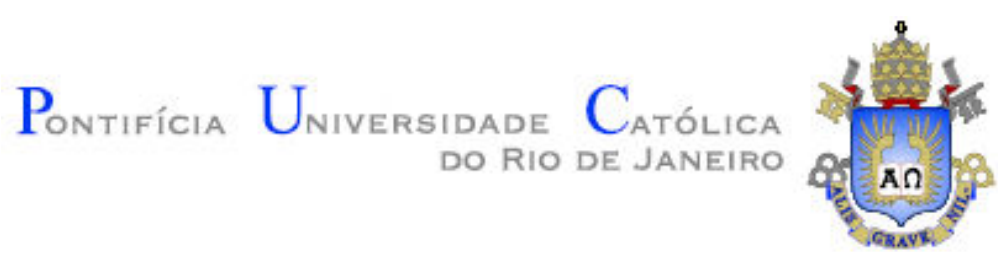

André Luiz Tenório Rezende

\title{
Análise Numérica da Bolha de Separação do Escoamento Turbulento sobre Placa Plana Fina Inclinada
}

Tese de Doutorado

Tese apresentada como requisito parcial para obtenção do título de Doutor pelo Programa de Pós-Graduação em Engenharia Mecânica da PUC-Rio.

Orientadora: Angela Ourivio Nieckele

Rio de Janeiro

Setembro 2009 


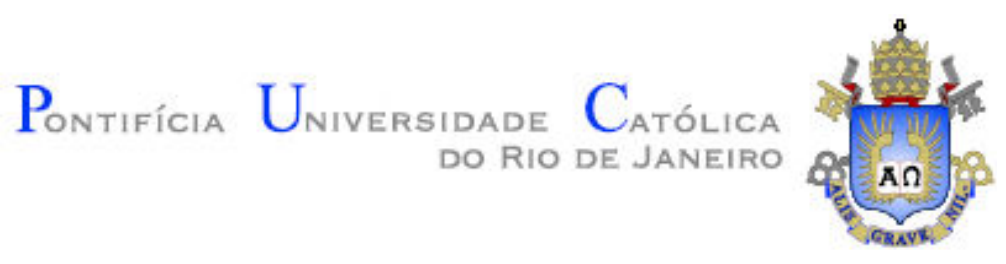

André Luiz Tenório Rezende

\title{
Análise Numérica da Bolha de Separação do Escoamento Turbulento sobre Placa Plana Fina Inclinada
}

\begin{abstract}
Tese apresentada como requisito parcial para obtenção do título de Doutor pelo Programa de Pós-Graduação em Engenharia Mecânica do Centro Técnico Científico da PUC-Rio. Aprovada pela Comissão Examinadora abaixo assinada.
\end{abstract}

Profa. Angela Ourivio Nieckele Orientadora Departamento de Engenharia Mecânica - PUC-Rio

Prof. José Diniz Mesquita Abrunhosa Instituto Militar de Engenharia

Prof. Marcelo José Colaço Universidade Federal do Rio de Janeiro

Prof. Marcelo José Santos de Lemos Instituto Tecnológico de Aeronáutica

Prof. Luiz Eduardo Bittencourt Sampaio Departamento de Engenharia Mecânica - PUC-Rio

Prof. José Eugenio Leal Coordenador Setorial do Centro Técnico Científico - PUC-Rio 
Todos os direitos reservados. É proibida a reprodução total ou parcial do trabalho sem autorização da universidade, do autor e do orientador.

\section{André Luiz Tenório Rezende}

Graduou-se em Engenharia Mecânica e de Armamento no Instituto Militar de Engenharia - IME, em 1996. Concluiu o Mestrado no IME em 2001, na área de Mecânica dos Sólidos estudando dinâmica de multicorpos rígidos aplicado à armamentos.

Ficha Catalográfica

Rezende, André Luiz Tenório

Análise numérica da bolha de separação do escoamento turbulento sobre placa plana fina inclinada / André Luiz Tenório Rezende ; orientador: Angela Ourivio Nieckele. -2009.

$$
263 \text { f. ; } 30 \mathrm{~cm}
$$

Tese (Doutorado em Engenharia Mecânica)Pontifícia Universidade Católica do Rio de Janeiro, Rio de Janeiro, 2009.

Inclui bibliografia

1. Engenharia mecânica - Teses. 2. Bolha de separação. 3. Turbulência. 4. RANS. 5. LES. 6. Placa Plana Fina Inclinada. I. Nieckele, Angela Ourivio. II. Pontifícia Universidade Católica do Rio de Janeiro. Departamento de Engenharia Mecânica. III. Título.

CDD: 621 


\section{Agradecimentos}

A Deus Pai, que é sinônimo de amor, união, paz, fé, sabedoria, saúde e perseverança.

A minha esposa Luciana, que é um exemplo de amor, mãe, dedicação e inteligência. Obrigado por tanta felicidade, cumplicidade e carinho em todos estes anos. Obrigado por tudo!

Ao meu filho Rafael, que espera há três anos para jogar bola comigo, mas ainda com coração puro sempre diz: "Papai, eu te amo!".

Aos meus pais Valmir e Denise, que se dedicaram incondicionalmente a minha formação intelectual e moral. Não há palavras que possam resumir a influência deles na minha vida, eles são os verdadeiros vitoriosos.

A minha sogra Dona Cida, avó maravilhosa que teve papel fundamental desde o nascimento do xodó Rafael, fornecendo tranqüilidade aos meus estudos e me tratando sempre como um verdadeiro filho.

Aos meus irmãos Maurício e Flávia, por todos os momentos felizes em família.

Aos meus avós, que sempre acreditaram nos meus estudos.

A minha orientadora Angela, pela amizade, suporte, ensinamento e fundamental orientação. Expresso minha admiração e orgulho por ter sido orientado por ela.

Ao Exército Brasileiro, "Braço Forte, Mão Amiga", que me acolheu como Oficial do Quadro de Engenheiros Militares e sempre acreditou no meu trabalho.

Ao meu atual chefe no IME, Tenente-Coronel Hamaoka, pela confiança e constante apoio, além de ser um verdadeiro amigo. A este militar dedico toda a minha lealdade.

Ao meu antigo chefe no CTEx, Coronel Correia Lima, pelos ensinamentos do verdadeiro profissional militar, servindo como inspiração de conduta do Oficial Engenheiro Militar. Além disso, o Coronel Correia Lima é um grande amigo. 
Ao meu compadre Capitão Vasconcellos, pelos 18 anos de amizade fraterna e que me presenteou com o meu lindo afilhado Luiz Henrique. Amizade boa é amizade de compadre!

Aos Oficiais do CTEx, os amigos fraternos Majores Eifler e Luis, com quem tive a honra de trabalhar em diversas campanhas de testes de armamentos, tornando o Grupo de Armamento e Munições o mais consistente possível.

Aos Oficiais professores do IME, os grandes amigos Majores Vieira Carneiro, Ricardo e Audrin e os Capitães Egydio Carvalho, Ricardo, Aldélio, Quinderé e Marcos José que me apoiaram sempre e em todos os momentos na minha jornada final no doutorado.

A todos os professores da Engenharia Mecânica do IME, que me receberam muito bem. Especialmente agradeço aos professores Francesco, Guedes e Colaço, além do Coronel Diniz que motivou a minha vinda para o IME.

Ao meu amigo e presidente, Coronel Júlio, pela manutenção da união, amizade e brincadeiras entre os membros da confraria da Engenharia Mecânica do IME. Pelo diário café da manhã com os Oficiais e professores e pelos almoços das quartas-feiras. Obrigado pelo acolhimento, preocupação e apoio.

Ao casal de amigos Cláudia e Luís André, que sempre estiveram ao meu lado, da Lú e do Rafa.

Ao meu melhor amigo na PUC, o tricolor Fernando. Começamos juntos, estamos terminando juntos, mas a amizade sempre continuará.

Ao amigo LuizEBS, pelos ensinamentos e ajuda em todas as fases da minha tese.

A todos aqueles que de alguma forma ajudaram na conclusão da minha tese.

Ao CNPq pelo apoio financeiro. 


\section{Resumo}

Rezende, André Luiz Tenório; Nieckele, Ângela Ourivio. Análise Numérica da Bolha de Separação do Escoamento Turbulento sobre Placa Plana Fina Inclinada. Rio de Janeiro, 2009. 263p. Tese de Doutorado Departamento de Engenharia Mecânica, Pontifícia Universidade Católica do Rio de Janeiro.

A estabilização de mísseis e projéteis é normalmente realizada através de aletas, que podem ser representadas por placas planas finas. $O$ escoamento sobre placas finas é de difícil previsão por apresentar diversos fenômenos, tais como transição da camada cisalhante para regime turbulento, recolamento, relaminarização e geração de bolhas primárias e secundárias. A proposta deste trabalho é analisar o escoamento turbulento ao longo de uma placa plana com pequeno ângulo de incidência, e ao mesmo tempo investigar o desempenho de diferentes modelos para a previsão da turbulência, empregando duas metodologias. A primeira é baseada nas Equações de Média de Reynolds (RANS), a qual requer um menor esforço computacional, por considerar um domínio bi-dimensional e regime permanente. Neste caso, três níveis de modelagens foram selecionados, os quais envolvem a solução de uma, duas e cinco equações diferenciais parciais, correspondendo aos modelos de SpalartAllmaras (SA), $\kappa-\omega$ Shear Stress Tensor (SST) e Reynolds Stress Model (RSM), respectivamente. No segundo enfoque, investigou-se o desempenho do modelo Smagorinsky Dinâmico, que é proveniente da metodologia da Simulação de Grandes Escalas (LES), a qual é tri-dimensional e transiente. Os resultados foram obtidos para número de Reynolds igual a 2,13 × $10^{5}$ e para três ângulos de incidência (um, três e cinco graus). A modelagem da turbulência foi validada através de comparação como dados numéricos e experimentais existentes na literatura. Os resultados obtidos mostraram que apesar do modelo RSM conseguir uma melhor previsão dos níveis de turbulência, o mesmo não é adequado para prever camadas cisalhantes livres. Já o modelo SA é muito difusivo, e não consegue prever adequadamente as tensões normais turbulentas, enquanto que o modelo SST foi capaz de prever razoavelmente bem a bolha de separação. Porém, apesar do custo bem superior, as previsões dos fenômenos provenientes da bolha de recirculação principal obtidas com a metodologia LES foram sensivelmente superiores e forneceram maior riqueza de informações que as apresentadas pelas soluções RANS.

\section{Palavras-chave}

Bolha de Separação, Turbulência, RANS, LES, Placa Plana Fina Inclinada. 


\section{Abstract}

Rezende, André Luiz Tenório; Nieckele, Ângela Ourivio (Advisor). Numerical Investigation of the Turbulent Flow Separation Bubble Over Inclined Thin Flat Plate. Rio de Janeiro, 2009. 263p. DSc. Thesis Departamento de Engenharia Mecânica, Pontifícia Universidade Católica do Rio de Janeiro.

Missiles and projectiles stabilization is usually accomplished through fins, which can be represented by thin flat plates. The flow field over thin plates is difficult to predict due to the existence of laminar-to-turbulent transition, boundary layer separation, leading edge bubble and reattachment. The purpose of this study is to analyze the flow over a thin flat plate, and at the same time, to investigate the performance of different models to predict turbulence, by employing two methodologies. The first one is based on the Reynolds Average Navier-Stokes Equations (RANS), which requires less computational effort, since it can be applied to a two-dimensional steady flow. In this case, three levels of modeling were employed, through the solution of one, two and five differential equations, corresponding to the Spalart-Allmaras (SA), $\kappa-\omega$ Shear Stress Tensor (SST) and Reynolds Stress Model (RSM) models, respectively. The second approach corresponds to the Large Eddy Simulation (LES) methodology, and the performance of the Dynamic Smagorinsky model was investigated. Results were obtained for Reynolds number equal to $2.13 \times 10^{5}$ and for three incidence angles (one, three and five degrees). The results were validated by comparing with available numerical and experimental data. It was shown that, in spite of predicting better turbulence levels, the RSM is not adequate to predict free shear layers. The SA model is too diffusive and it fails to predict the normal stresses, while the SST is capable of predicting the separation bubble with reasonable accuracy. However, in spite of the larger cost, the long separation bubble predictions obtained with the LES methodology were substantial superior and more complete than RANS solutions.

\section{Keywords}

Separation Bubble, Turbulence, LES, RANS, Inclined Thin Flat Plate 


\title{
Nomenclatura
}

\author{
DES Modelo Híbrido LES/RANS \\ DNS Simulação Numérica Direta \\ MVF Método dos Volumes Finitos \\ LES Simulação de Grandes Escalas \\ $f$-LES Modelo de forçamento sub-malha \\ LDV Velocimetria a Laser com efeito Doppler \\ SIMPLE Algoritmo de solução do acoplamento velocidade-pressão \\ PISO Algoritmo de solução do acoplamento velocidade-pressão \\ QUICK Esquema de discretização espacial de segunda ordem \\ RANS Abordagem da turbulência, baseada em média de Reynolds \\ URANS RANS transiente \\ SGS Escala sub-malha \\ UPWIND Esquema de discretização espacial de primeira ordem
}




\section{Lista de Símbolos}

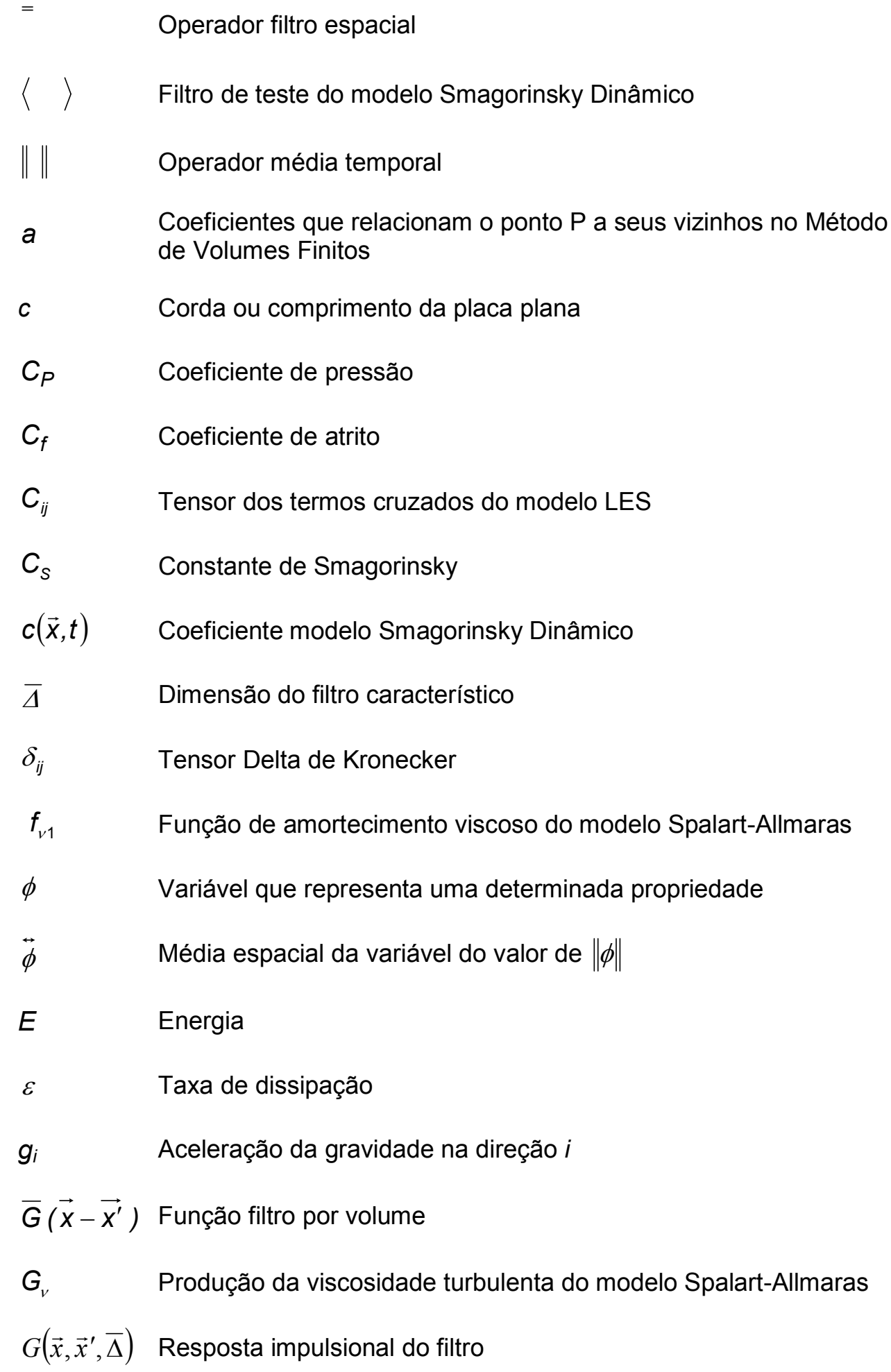




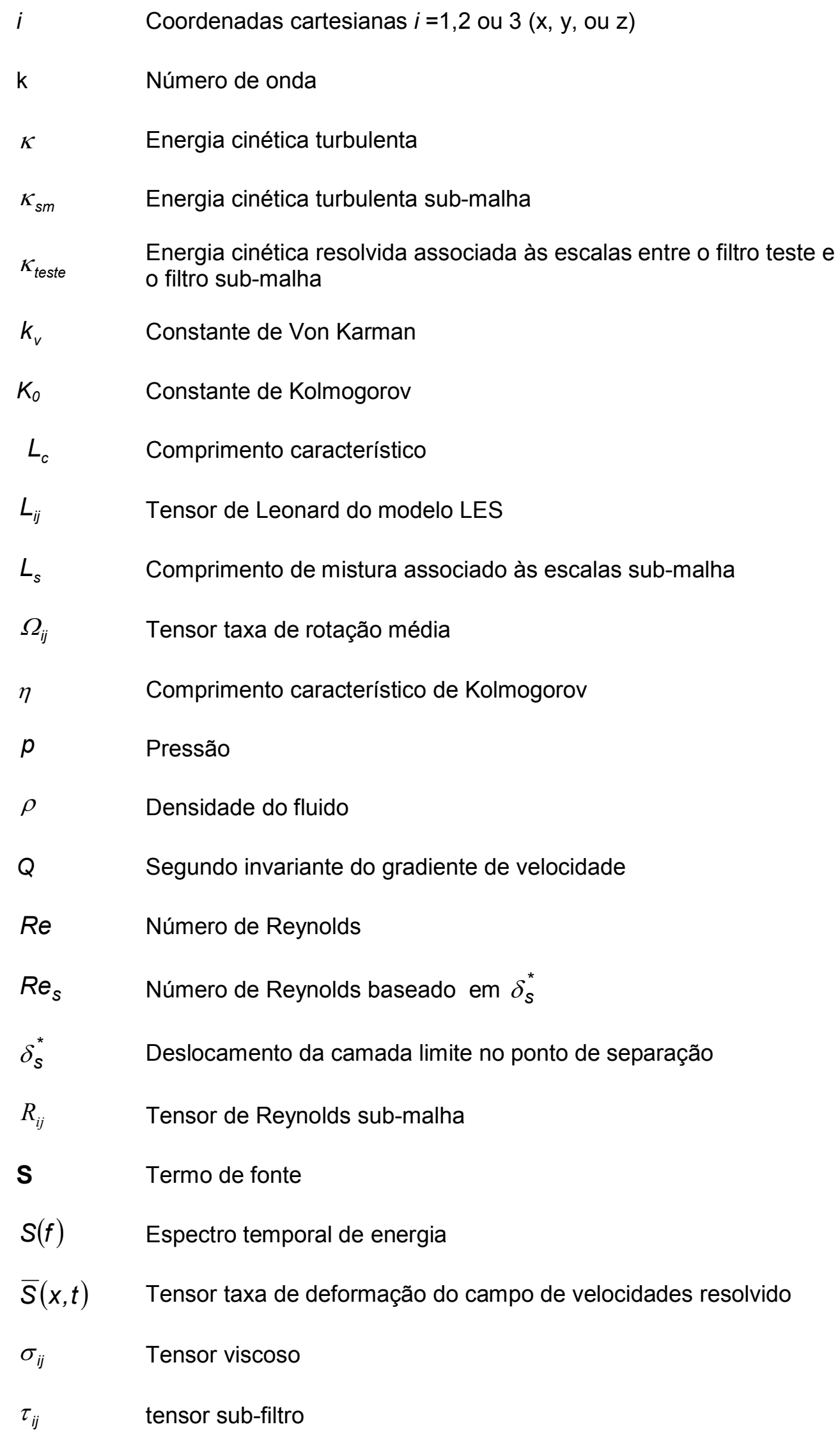




\begin{tabular}{|c|c|}
\hline$t$ & Variável de tempo \\
\hline$\Gamma$ & Coeficiente de difusão \\
\hline$u_{i}$ & Componente da velocidade na direção $i(x, y$, ou $z)$ \\
\hline$u_{\tau}$ & Velocidade de atrito \\
\hline $\bar{u}_{i}$ & Componentes da velocidade média na direção $i$. Modelos RANS \\
\hline$u_{i}^{\prime}$ & Flutuação da velocidade na direção $i$ \\
\hline$\overline{\overline{u_{i}}}$ & Componentes da velocidade filtrada na direção $i$. Modelos LES \\
\hline$u_{i}^{\prime \prime}$ & Parcela da velocidade na escala sub-malha. Modelos LES \\
\hline$U_{\infty}$ & Magnitude da velocidade na corrente livre \\
\hline$V_{c}$ & Velocidade característica \\
\hline$\forall$ & Volume de discretização do modelo de Smagorinsky \\
\hline$\mu$ & Viscosidade absoluta ou viscosidade dinâmica \\
\hline$\mu_{t}$ & Viscosidade absoluta turbulenta \\
\hline$\mu_{\text {ef }}$ & Viscosidade efetiva \\
\hline$\tilde{v}$ & Variável auxiliar do modelo Spalart-Allmaras \\
\hline$v$ & Viscosidade cinemática \\
\hline$v_{t}$ & Viscosidade turbulenta \\
\hline$v_{S M}$ & Viscosidade turbulenta sub-malha \\
\hline$x, y, z$ & $\begin{array}{l}\text { Coordenadas cartesianas: } x \text {, a direção longitudinal à placa; y, a } \\
\text { direção vertical à placa; } z \text {, a direção da envergadura da placa }\end{array}$ \\
\hline$X_{R}$ & Comprimento de recolamento da bolha primária \\
\hline$X_{S}$ & Comprimento de recolamento da bolha secundária \\
\hline$y^{+}$ & Distância adimensional para parede \\
\hline$Y_{v}$ & Destruição da viscosidade turbulenta do modelo Spalart-Allmaras \\
\hline
\end{tabular}




\section{Sumário}

1 Introdução 26

1.1. Objetivo e Escopo do Trabalho 31

1.2. Organização do Trabalho 33

2 Revisão Bibliográfica $\quad 34$

2.1. Bolha de Separação Laminar (Bolha Curta) 34

2.2. Bolha de Separação Longa 40

2.3. Importância e Principais Abordagens Numéricas da Turbulência 44

2.3.1. Aspectos Gerais dos Modelos de Turbulência 47

2.3.2. Aplicação dos Modelos de Turbulência à Placa Plana em Estudo 49

3 Modelagem da Turbulência $\quad 51$

3.1. Equações de Governo 53

3.2. Equações de Médias de Reynolds - RANS 54

3.2.1. Hipótese de Boussinesq 56

3.2.2. Equações de Transporte do Tensor de Reynolds - RSM 66

3.3. Simulação de Grandes Escalas - LES

3.3.1. Modelagem Sub-Malha no LES 80

4 Método Numérico $\quad 87$

4.1. Considerações sobre o Método de Volumes Finitos 88

4.2. Discretização e Solução da Equação Geral de Transporte 89

4.2.1. Discretização Espacial 91

4.2.2. Discretização Temporal 97

4.3. Discretização das Equações de Navier-Stokes 98

4.3.1. Acoplamento Pressão-Velocidade 100

4.4. Método para Solução do Sistema Algébrico 102

4.4.1. Critério de Convergência 103

4.5. Características do Algoritmo Utilizado na Solução do Escoamento 103

5 Ângulo de Incidência de $\alpha=1^{0} \quad 106$

5.1. Dados Experimentais 107 
5.2. Domínio Computacional e Condições de Contorno 109

5.2.1. Condições de Contorno 110

5.3. Modelos RANS 111

5.3.1. Campo de Velocidade 112

5.3.2. Estatísticas de Segunda Ordem 122

5.3.3 Coeficiente de Atrito e de Pressão 132

5.4. Simulação de Grandes Escalas (LES) 135

5.4.1. Campo de Velocidade 143

5.4.2. Estatísticas de Segunda Ordem 147

5.4.3. Distribuição de Pressão 157

5.4.4. Características do Escoamento Turbulento 158

6 Ângulo de Incidência de $\alpha=3^{0} \quad 168$

6.1. Campo de Velocidade 174

6.2. Estatísticas de Segunda Ordem 181

6.3. Coeficiente de Pressão 187

6.4 Estruturas Coerentes 189

7 Ângulo de Incidência de $\alpha=5^{0} \quad 193$

7.1. Campo de Velocidade 196

7.2. Estatísticas de Segunda Ordem 206

7.3. Coeficiente de Pressão 216

7.4. Estruturas Coerentes 218

8 Comentários Finais 222

8.1. Conclusão 223

8.2. Recomendações 228

$\begin{array}{ll}\text { Referências Bibliográficas } & 230\end{array}$

APÊNDICE A Modelos Sub-Malha 243

A.1. Modelo Sub-Malha de uma Equação 243

A.2. Modelo de Forçamento Sub-Malha (f-LES) 244 
APÊNDICE B - Domínio Computacional e Malha 247

B.1. Simulações RANS 247

B.2. Simulações LES 255

B.2.1. Simulação LES para o Ângulo de Ataque $\alpha=3^{0}$

APÊNDICE C Influência da Intensidade de Turbulência na Entrada 262 


\section{Lista de figuras}

Figura 1.1 - Aletas fixas para estabilização de projéteis.

Figura 2.1 - Separação da camada limite e fenômeno da estolagem.

Figura 2.2 - Bolha curta laminar (Horton, 1969).

Figura 2.3 - Bolha curta laminar (Crompton, 2001).

Figura 2.4 - Esquema do escoamento sobre uma placa plana fina inclinada.

Figura 2.5 - Estrutura interna da bolha (Collie, 2005).

Figura 2.6 - Esquema do escoamento sobre uma placa plana fina inclinada.

Figura 2.7 - Visualização da turbulência por Leonardo da Vinci.

Figura 3.1 - Grau de modelagem e custo computacional dos modelos de turbulência.

Figura 3.2 - Espectro de energia.

Figura 4.1 - Exemplo de Volume de controle para mostrar

a discretização da equação de transporte de um escalar.

Figura 4.2 -Volume de Controle Unidimensional.

Figura 4.3 - Diagrama de Variáveis Normalizadas (Choi et al., 1995).

Figura 4.4 - Diagrama de Variáveis Normalizadas (Choi et al., 1995).

Figura 4.5 - Seqüência de malhas para o método Multigrid.

Figura 4.6 - Algoritmo utilizado na solução do escoamento incompressível proposto.

Figura 5.1 - Dimensões da placa utilizada por Crompton.

Figura 5.2 - Estações de medição sobre a placa e posição de recolamento para $\alpha=1^{0}$.

Figura 5.3 - Detalhes do domínio computacional utilizado neste trabalho. 
Figura 5.4 - Contornos de velocidade média $u / U_{\infty}$ para $\alpha=1^{0}$.

Modelos RANS.

Figura 5.5 - Evolução dos perfis de velocidade $u / U_{\infty}$.

Modelos RANS. $\alpha=1^{0}$

Figura 5.6 - Perfis de velocidade $u / U_{\infty}$ para as estações

antes do recolamento. Modelos RANS. $\alpha=1^{0}$

Figura 5.7 - Detalhe do perfil de velocidade $u / U_{\infty}$ para a

estação $1(x / c=0,031)$. Modelos RANS. $\alpha=1^{0}$

Figura 5.8 - Perfis de $u / U_{\infty}$ para as estações localizadas

após o recolamento. Modelos RANS. $\alpha=1^{0}$

Figura 5.9-Perfil de velocidade para $u^{+}$em $x / c=0,875$.

Modelos RANS. $\alpha=1^{0}$

Figura 5.10 - Linhas de corrente para os modelos RANS. $\alpha=1^{0}$

Figura 5.11 - Perfis de $\overline{u^{\prime} u^{\prime}} / U_{\infty}^{2}$ : estações localizadas

antes do recolamento. Modelos RANS. $\alpha=1^{0}$

Figura 5.12 - Perfis de $\overline{u^{\prime} u^{1}} / u_{\infty}^{2}$ : estações localizadas após

o recolamento. Modelos RANS. $\alpha=1^{0}$

Figura 5.13 - Perfis de $\overline{v^{\prime} v^{\prime}} / U_{\infty}$ : estações localizadas antes

do recolamento. Modelos RANS. $\alpha=1^{0}$

Figura 5.14 - Tensão normal $\overline{v^{\prime} v^{\prime}} / U_{\infty}$ : estações localizadas

após o recolamento. Modelos RANS. $\alpha=1^{0}$

Figura 5.15 - Perfis de $\overline{u^{\prime} v^{\prime}} / U_{\infty}$ : estações localizadas antes

do recolamento. Modelos RANS. $\alpha=1^{0}$

Figura 5.16 - Perfis de $\overline{u^{\prime} v^{\prime}} / U_{\infty}$ : estações localizadas após

o recolamento. Modelos RANS. $\alpha=1^{0}$

Figura 5.17 - Contornos das tensões de Reynolds -

modelo Spalart-Allmaras $\left(\alpha=1^{\circ}\right)$.

Figura 5.18 - Contornos das tensões de Reynolds -

modelo SST $\left(\alpha=1^{0}\right)$. 
Figura 5.19 - Contornos das tensões de Reynolds -

modelo RSM $\left(\alpha=1^{\circ}\right)$.

Figura 5.20 - Coeficiente de atrito superficial para $\alpha=1^{0}$.

Modelos RANS.

Figura 5.21 - Coeficiente de pressão para $\alpha=1^{0}$.

Figura 5.22 - Posicionamento das sondas sobre a placa. $\alpha=1^{0}$.

Figura 5.23 - Evolução da velocidade no tempo na direção $x$ -

sondas P1, P2 e P3. $\alpha=1^{0}$

Figura 5.24 - Evolução da velocidade no tempo na direção $y$ -

sondas P1, P2 e P3. $\alpha=1^{0}$

Figura 5.25 - Evolução da velocidade no tempo na direção $Z$ -

sondas P1, P2 e P3. $\alpha=1^{0}$

Figura 5.26 - Evolução da velocidade no tempo na direção $x$ sondas P4, P5 e P6. $\alpha=1^{0}$

Figura 5.27 - Evolução da velocidade no tempo na direção $x$ sondas P7, P8 e P9.

Figura 5.28 - Evolução da velocidade no tempo na direção $x$ sondas P10, P11 e P12.

Figura 5.29 - Evolução da velocidade no tempo na direção $x$ sondas $\mathrm{P} 13, \mathrm{P} 14$ e P15.

Figura 5.30 - Perfis de velocidade $u / U_{\infty}$ : estações localizadas antes do recolamento. Comparação entre LES e RANS. $\alpha=1^{0}$

Figura 5.31 - Detalhe dos perfis de velocidade $u / U_{\infty}$ para $x / c=0,031$ e $x / c=0,125$. Comparação entre LES e RANS. $\alpha=1^{0}$

Figura 5.32 - Perfis de velocidade $u / U_{\infty}$ : estações localizadas após o recolamento. Comparação entre LES e RANS. $\alpha=1^{0}$

Figura 5.33 - Linhas de corrente para o modelo

LES Dinâmico. $\alpha=1^{0}$

Figura 5.34 - Perfis de $u^{\prime} u^{\prime} / U_{\infty}^{2}$ : estações localizadas após

o recolamento. Comparação entre LES e RANS. $\alpha=1^{0}$ 
Figura 5.35 - Perfis de $u^{\prime} u^{\prime} / U_{\infty}^{2}$ : estações localizadas após o recolamento. Comparação entre LES e RANS. $\alpha=1^{0}$

Figura 5.36 - Contornos do $u_{r m s} / U_{\infty}(\%)$. A linha branca representa $u / U_{\infty}=0$. Ângulo de atque $\alpha=1^{0}$

Figura 5.37 - Perfis de $v^{\prime} v^{\prime} / U_{\infty}^{2}$ : primeiras quatro estações.

Comparação entre LES e RANS. $\alpha=1^{0}$

Figura 5.38 - Contornos de $v^{\prime} v^{\prime} / U_{\infty}^{2}$ : LES Dinâmico, $\alpha=1^{0}$.

(a) Placa inteira (b) Região da bolha de separação

Figura 5.39 - Perfis de $u^{\prime} v^{\prime} / U_{\infty}^{2}$ : primeiras quatro estações.

Comparação entre LES e RANS. $\alpha=1^{0}$

Figura 5.40 - Contornos de $u^{\prime} v^{\prime} / U_{\infty}^{2}$. LES Dinâmico, $\alpha=1^{0}$.

(a) Placa inteira (b) Região da bolha de separação

Figura 5.41 - Perfis de $w^{\prime} w^{\prime} / U_{\infty}^{2}$ : primeiras quatro estações

(LES Dinâmico). $\alpha=1^{0}$.

Figura 5.42 - Contornos de $w^{\prime} w^{\prime} / U_{\infty}^{2}$ - LES Dinâmico, $\alpha=1^{0}$.

(a) Placa inteira (b) Região da bolha de separação

Figura 5.43 - Contornos de $w^{\prime} w^{\prime} / U_{\infty}^{2}$ - RSM, $\alpha=1^{0}$.

(a) Placa inteira (b) Região da bolha de separação

Figura 5.44 - Coeficiente de pressão nas simulações LES

para $\alpha=1^{0}$.

Figura 5.45 - Contornos do coeficiente de pressão nas

simulações LES e SST $\left(\alpha=1^{0}\right)$.

Figura 5.46 - Espectros temporais e histogramas para

os três componentes da velocidade na sonda P1.

Figura 5.47 - Espectro temporal e histograma para o

componente $x$ da velocidade na sonda P4.

Figura 5.48 - Espectro temporal e histograma para o

componente $x$ da velocidade na sonda P7.

Figura 5.49 - Espectro temporal e histograma para o

componente $\mathrm{x}$ da velocidade na sonda P10. 
Figura 5.50 - Espectro temporal e histograma para o

componente $x$ da velocidade na sonda P13.

Figura 5.51 - Contornos da razão entra as viscosidades sub-malha e molecular (toda a placa e região da bolha). LES Dinâmico, $\alpha=1^{0}$.

(a) Placa inteira (b) Região da bolha de separação

Figura 5.52 - Estruturas coerentes sob diferentes critérios.

Figura 5.53 - Estruturas vorticais coerentes através do critério $Q$.

Figura 6.1 - Detalhes do domínio computacional para o caso

LES Dinâmico em $\alpha=3^{0}$.

Figura 6.2 - Posição das sondas sobre a placa. $\alpha=3^{0}$.

Figura 6.3 - Evolução da velocidade no tempo -

sonda P1. $\alpha=3^{0}$

Figura 6.4 - Evolução da velocidade $x$ no tempo. $\alpha=3^{0}$.

Figura 6.5 - Contornos de velocidade $u / U_{\infty}$ para $\alpha=3^{0}$.

Modelos RANS e LES.

Figura 6.6 - Evolução dos perfis de velocidade $u / U_{\infty}$.

Modelos RANS e LES. $\alpha=3^{0}$

Figura 6.7 - Perfis de velocidade $u / U_{\infty}$ : estações localizadas

antes do recolamento. Comparação entre LES e RANS. $\alpha=3^{0}$

Figura 6.8 - Detalhe dos perfis de velocidade $u / U_{\infty}$ para $x / c=0,031$.

Comparação entre LES e RANS. $\alpha=3^{0}$.

Figura 6.9 - Linhas de corrente. Comparação entre

LES e RANS. $\alpha=3^{\circ}$.

Figura 6.10 - Perfis de velocidade $v / U_{\infty}$. Comparação entre

LES e RANS. $\alpha=3^{0}$.

Figura 6.11 - Perfis de $u^{\prime} u^{\prime} / U_{\infty}^{2}$ : estações localizadas antes

do recolamento. Comparação entre LES e RANS. $\alpha=3^{\circ}$.

Figura 6.12 - Perfis de $v^{\prime} v^{\prime} / U_{\infty}^{2}$ : estações localizadas antes do recolamento.

Comparação entre LES e RANS. $\alpha=3^{0}$. 
Figura 6.13 - Perfis de energia cinética turbulenta: estações no interior da bolha. Comparação entre LES e RANS. $\alpha=3^{0}$.

Figura 6.14 - Perfis de $u^{\prime} v^{\prime} / U_{\infty}^{2}$ : estações localizadas antes do recolamento.

Comparação entre LES e RANS. $\alpha=3^{0}$.

Figura 6.15 - Contornos das grandezas de segunda ordem -

SST. $\alpha=3^{0}$.

Figura 6.16 - Contornos das grandezas de segunda ordem Spalart Allmaras. $\alpha=3^{0}$.

Figura 6.17 - Contornos das grandezas de segunda ordem LES Dinâmico. $\alpha=3^{0}$.

Figura 6.18 - Coeficiente de pressão - $C_{P}$. Comparação entre

LES e RANS. $\alpha=3^{0}$

Figura 6.19 - Estruturas coerentes analisadas através

do critério $Q$. $\alpha=3^{0}$

Figura 6.20 - Estruturas coerentes através do critério

$Q\left(Q c / U_{\infty}=160\right)$ coloridas pela intensidade da magnitude

da vorticidade $\left(|\omega| c / U_{\infty}\right) \cdot \alpha=3^{0}$

Figura 6.21 - Estruturas coerentes sob o critério da região de alto módulo da vorticidade. Valor normalizado:

$|\omega| c / U_{\infty}=160 . \alpha=3^{0}$

Figura 6.22 - Estruturas coerentes sob o critério da região

de baixa pressão total. Valor normalizado:

$P /\left(\rho U_{\infty}^{2}\right)=-0,38 \cdot \alpha=3^{0}$

Figura 7.1 - Localização do ponto de recolamento para $\alpha=5^{0}$.

Figura 7.2 - Contornos Contornos de velocidade $u / U_{\infty}$ para $\alpha=5^{\circ}$. Modelos RANS e LES.

Figura 7.3 - Evolução dos perfis de velocidade $u / U_{\infty}$ para $\alpha=5^{\circ}$.

Figura 7.4 - Detalhe dos perfis de velocidade $u / U_{\infty}$ para

$x / c=0,031$. Comparação entre LES e RANS. $\alpha=5^{0}$. 
Figura 7.5 - Perfis de velocidade $u / U_{\infty}$. Comparação entre

LES e RANS. $\alpha=5^{0}$

Figura 7.6 - Linhas de corrente - Modelo LES Dinâmico

com detalhe da segunda bolha de recirculação $\left(\alpha=5^{\circ}\right)$.

Figura 7.7 - Linhas de corrente - Modelo SST com detalhe da segunda bolha de recirculação $\left(\alpha=5^{\circ}\right)$.

Figura 7.8 - Linhas de corrente - Modelo

Spalart-Allmaras $\left(\alpha=5^{0}\right)$.

Figura 7.9 - Perfis de velocidade $v / U_{\infty}$. Comparação entre

LES e RANS. $\alpha=5^{\circ}$.

Figura 7.10 - Contornos do módulo da velocidade instantânea

normalizado por $U_{\infty} \cdot \alpha=5^{0}$.

Figura 7.11 - Perfis de $u^{\prime} u^{\prime} / U_{\infty}^{2}$. Comparação entre

LES e RANS. $\alpha=5^{\circ}$.

Figura 7.12 - Perfis de energia cinética turbulenta.

Comparação entre LES e RANS. $\alpha=5^{\circ}$.

Figura 7.13 - Perfis de $v^{\prime} v^{\prime} / U_{\infty}^{2}$ : Comparação entre

LES e RANS. $\alpha=5^{\circ}$.

Figura 7.14 - Perfis de $u^{\prime} v^{\prime} / U_{\infty}^{2}$. Comparação entre

LES e RANS. $\alpha=5^{\circ}$.

Figura 7.15 - Contornos das estatísticas de segunda

ordem - Spalart Allmaras. $\alpha=5^{\circ}$.

Figura 7.16 - Contornos das estatísticas de segunda

ordem - SST. $\alpha=5^{\circ}$

Figura 7.17 - Contornos das estatísticas de segunda

ordem - LES Dinâmico. $\alpha=5^{\circ}$.

Figura 7.18 - Coeficiente de pressão - $C_{P}$. Comparação entre

LES e RANS. $\alpha=5^{\circ}$.

Figura 7.19 - Coeficiente de pressão - $C_{P}$. Comparação entre ângulos de incidência. LES Dinâmico. 
Figura 7.20 - Estruturas coerentes analisadas através do

critério $Q . \alpha=5^{\circ}$.

Figura 7.21 - Estruturas coerentes através do critério

$Q\left(Q c / U_{\infty}=160\right)$ coloridas pela intensidade da

magnitude da vorticidade $\left(|\omega| c / U_{\infty}\right) . \alpha=5^{0}$.

220

Figura 7.22 - Região de alto módulo da vorticidade.

Valor normalizado: $|\omega| c / U_{\infty}=160 . \alpha=5^{\circ}$.

Figura B.1 - Detalhes do domínio computacional de

Sampaio (2006).

Figura B.2 - Detalhes do domínio computacional utilizado neste trabalho.

Figura B.3 - Domínio computacional dividido em blocos e suas dimensões.

Figura B.4 - Detalhes da malha 150K utilizada na discretização da placa

Figura B.5 - Comparação entre as malhas através de $\bar{u}$

(Modelo SST - $\alpha=1^{0}$ ).

Figura B.6 - Comparação entre as malhas através de $\overline{u^{\prime} u^{\prime}}$

(Modelo SST - $\alpha=1^{0}$ ).

Figura B.7 - Comparação entre as malhas através de $C_{P}$

(Modelo SST - $\alpha=1^{0}$ ).

Figura B.8 - Valores de $y^{+}$para os modelos RANS. $\alpha=1^{0}$.

Figura B.9 - Valores de $y^{+}$para os modelos RANS $\left(\alpha=3^{0}\right)$.

Figura B.10 - Valores de $y^{+}$para os modelos RANS $\left(\alpha=5^{\circ}\right)$.

Figura B.11 - Valores instantâneos de $y^{+} \cong 1$.

Figura B.12 - Valores instantâneos de $y^{+}$sobre a placa $\left(\alpha=5^{0}\right)$.

Figura B.13 - Detalhes do domínio computacional para o caso

LES Dinâmico em $\alpha=3^{0}$. 
Figura B.14 - Detalhes da malha utilizada na discretização da placa plana $\left(\alpha=3^{0}\right)$. (a) Plano x-y. Domínio completo, para $\alpha=3^{0}$

(b) Malha na região da placa, para $\alpha=3^{0}$ (c) Malha na região da ponta de faca da placa, para $\alpha=3^{0}$

Figura B.15 - Valores instantâneos de $y^{+}$sobre a placa $\left(\alpha=3^{0}\right)$.

Figura B.16 - Comparação entre as malhas 2,4M e 2,9M $\left(\alpha=3^{0}\right) . \quad 261$

Figura C.1- Avaliação da condição de contorno da

intensidade turbulenta na entrada . 


\section{Lista de tabelas}

Tabela 3.1 - Constantes Empíricas do Modelo Spalart Allmaras 62

Tabela 3.2 - Coeficientes do modelo SST 65

Tabela 3.3 - Coeficientes do modelo RSM para equação de $\varepsilon \quad 70$

Tabela 3.4 - Coeficientes para tratamento do modelo RSM

$\begin{array}{ll}\text { próximo à parede } & 77\end{array}$

Tabela 4.1 - Algoritmo utilizado na solução do escoamento

$\begin{array}{ll}\text { incompressível proposto. } & 104\end{array}$

Tabela 5.1 - Posição das estações de medição dos perfis de velocidade. 108

Tabela 5.2 - Comprimentos de recolamento normalizados

$\left(X_{R}\right)$ e os respectivos erros.

Tabela 5.3 - Coordenadas normalizadas das sondas

sobre a placa $\left(\alpha=1^{0}\right)$.

Tabela 5.4 - Coordenadas normalizadas das sondas na

esteira da placa $\left(\alpha=1^{0}\right)$.

Tabela 5.5 - Comprimentos de recolamento $X_{R}$ e $X_{S}$ para os

modelos LES.

Tabela 6.1 - Coordenadas normalizadas das sondas sobre

a placa $\left(\alpha=3^{0}\right)$.

Tabela 6.2 - Comprimentos de recolamento normalizados

$X_{R}$ para $\alpha=3^{0}$.

Tabela 7.1 - Comprimentos de recolamento normalizados

$X_{R}$ e $X_{S}$ para $\alpha=5^{0}$.

Tabela B.1 - Subdivisões da malha 330K para os blocos

da Figura B.3 .

Tabela B.2 - Subdivisões da malha $150 \mathrm{~K}$ para os blocos

da Figura B.3.

Tabela B.3 - Subdivisões da malha com a placa inclinada para os blocos da Figura B.2. 